\title{
Estimating soil aggregate size distribution from images using pattern spectra
}

\author{
Petra Bosilja,b,*, Iain Gould ${ }^{\mathrm{b}}$, Tom Duckett ${ }^{\mathrm{a}}$, Grzegorz Cielniak ${ }^{\mathrm{a}}$ \\ ${ }^{a}$ Lincoln Centre for Autonomous Systems, School of Computer Science, University of \\ Lincoln, Brayford Campus, LN6 7TS Lincoln, UK \\ ${ }^{b}$ Lincoln Institute for Agri-food Technology, University of Lincoln, Riseholme Park, LN2 \\ $2 L G$ Lincoln, UK
}

\begin{abstract}
A method for quantifying aggregate size distribution from the images of soil samples is introduced. Knowledge of soil aggregate size distribution can help to inform soil management practices for the sustainable growth of crops. While current in-field approaches are mostly subjective, obtaining quantifiable results in a laboratory is labour- and time-intensive. Our goal is to develop an imaging technique for quantitative analysis of soil aggregate size distribution, which could provide the basis of a tool for rapid assessment of soil structure. The prediction accuracy of pattern spectra descriptors based on hierarchical representations from attribute morphology are analysed, as well as the impact of using images of different quality and scales. The method is able to handle greater sample complexity than the previous approaches, while working with smaller samples sizes that are easier to handle. The results show promise for size analysis of soils with larger structures, and minimal sample preparation, as typical of soil assessment in agriculture.
\end{abstract}

Keywords: pattern spectra, component trees, soil aggregate analysis

\footnotetext{
*Corresponding author: Tel.: +44 (0)1522 83 5378;

Email address: pbosilj@lincoln.ac.uk (Petra Bosilj)
} 


\section{Introduction and motivation}

Soil structure concerns the physical arrangement of a soil, which provides an environment to provide plants access to water, air and nutrients, and a suitable medium for root development (Bronick \& Lal, 2005). Adequate soil structure is

5 fundamental for the sustainable growth of crops, and can contribute to reducing the environmental impact of agriculture. Hence, robust and accurate methods to measure soil structure are important tools for informing soil management decisions.

Soil aggregates constitute a key structural unit of the soil, and are composites of sand, silt, clay (primary particles), organic matter and pore space. The strength of soil structural coherence depends on the binding forces between these materials, which are often a function of the soil's biological activity (Ashman, Hallett \& Brookes, 2003, Czarnes, Hallett, Bengough \& Young, 2000). As such, assessments of soil aggregates provide information not only on soil physical 15 structure, but also on the overall "health" of a soil, and indicate the potential of a soil to sustain vital ecosystem functions like crop growth, carbon sequestration and water regulation (Allen, Singh \& Dalal, 2011). A soil ped is a larger structural unit, which can be broken down into aggregates, whilst a soil clod is a larger, more angular structural unit that has undergone disturbance, which often does not break down into stable soil aggregates. Together, the arrangement and distribution of aggregates, peds and clods constitute the structural arrangement of a soil.

Several laboratory techniques have been developed to assess properties of soil size distribution. These methods may look at the stability of aggregate cohesion and/or the size distribution after aggregate breakdown (Beare \& Bruce, 1993 Le Bissonnais, 1996). They usually employ dry and wet sieving techniques, measuring the soil retained on sieve stacks with decreasing mesh sizes. Although such techniques, and subsequent developments, are routinely used in academic soil research, the timescales and relatively high labour input needed for size distribution analysis may explain the limited adoption of such processes 
in commercial agricultural laboratories. In-field assessments of soil structure, such as the visual evaluation of soil structure (VESS) or visual soil assessment (VSA) tests (Shepherd, 2009, Ball, Batey \& Munkholm, 2007) amongst others, are based on the size distribution of soil aggregate, ped or clod following in-field breakdown. Such methods offer a more rapid assessment of soil structure for practitioners, but they rely on simplified scoring and categorisation, for example a score of Sq1 (friable) to Sq5 (compact) for the VESS test, and a degree of subjectivity between assessments.

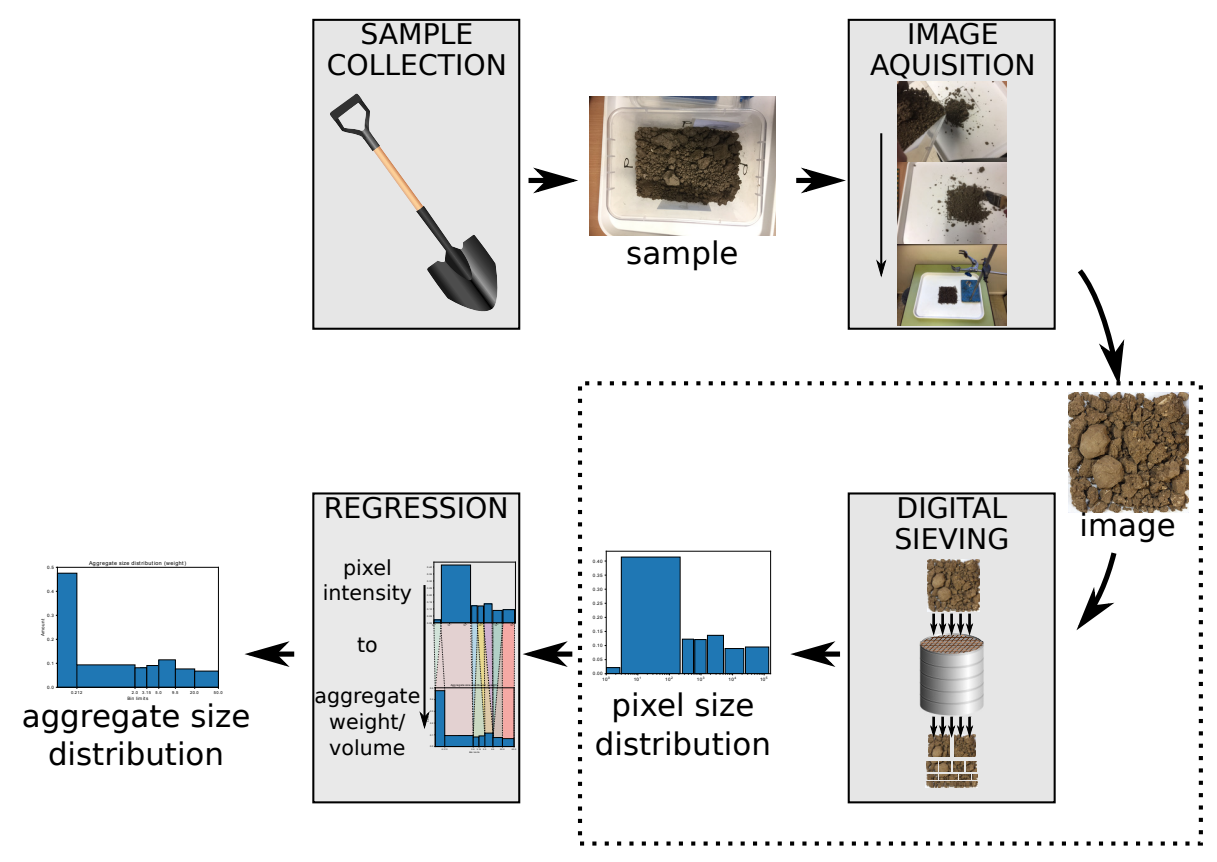

Figure 1: System overview.

Developments in applying image analysis to soil characterisation, such as aggregate distribution, could provide a solution to some of these limitations, by enhancing the efficiency and repeatability of quantitative in-field analysis without needing an expert practitioner (Aitkenhead, Donnelly, Coull \& Gwatkin, 2016). They would also be of particular interest when performing the assessment of soil structure in hostile environments such as on the Martian surface

45 (Karunatillake et al. 2014), where the physical samples are typically inaccessible 
and the analysis has to rely on imaging data. This paper proposes an automatic pipeline for measuring soil aggregate size distribution from images of soil samples, shown in Fig. 1. Soil samples from a depth of 0 to $200 \mathrm{~mm}$ were collected from a range of soil types under arable fields. This work focuses on replacing physical sieving with the digital sieving process, with the corresponding parts of the pipeline indicated in Fig. 1. This physical process closely matches the algorithmic process of granulometries (Matheron, 1975, Breen \& Jones, 1996) and pattern spectra (Maragos, 1989), where the image is filtered with a succession of openings of increasing sizes, often also described as sieving. The classical pattern spectra based on structuring element (SE) morphology are compared to their attribute morphology counterparts, which are rotation invariant and are less sensitive to noise (Urbach, Roerdink \& Wilkinson, 2007). Pattern spectra can be interpreted as histogram representations of the image component size distribution, which are then mapped to soil aggregate size distribution in terms of either mass or volume by a trained regression model.

While the first efficient implementations of granulometries and pattern spectra relied on the max-tree hierarchy (Salembier, Oliveras \& Garrido, 1998), this work investigates the ability of pattern spectra to capture the soil aggregate size distribution when calculated on both types of image hierarchies: inclusion trees (Salembier et al., 1998, Monasse \& Guichard, 2000), which are extremaoriented, and partitioning trees (Soille, 2007, 2008), which capture intermediate level regions. The contributions to knowledge of this work are:

- trained regression models are developed able to predict the measured soil aggregate size distributions from images in terms of mass and volume,

- the most suitable hierarchical representation for calculating attribute pattern spectra is identified and the performance is additionally compared to the classical SE spectra, and

- A dataset is published containing RGB images of soil samples at different scales, captured with both professional and amateur cameras, together 
with the results of associated manual soil aggregate size distribution analysis in terms of both mass and volume ${ }^{1}$.

The proposed pipeline allows a direct quantification of the soil aggregate size distribution, rather than just a re-identification of samples as in Bosilj, Gould, Duckett \& Cielniak, 2019), and could potentially be used in the development of ${ }_{80}$ an in-field system for automated soil analysis.

\section{Related Work}

Some of the earliest image processing techniques applied to estimation of aggregate size distribution focused on segmenting images of non-overlapping coarse aggregates ( $3 \mathrm{~mm}$ to $63 \mathrm{~mm}$ ) (Mora, Kwan \& Chan, 1998). An automated tool for measuring the grain size distribution of gravels from digital photographs 口was developed by Graham, Rice \& Reid (2005) and improved by Detert \& Weitbrecht (2012) based on analysing a number of segmentation-based techniques for overlapping particles of coarse-grained sediments, including those based on top-hat and watershed morphological operations (Graham, Reid \& Rice, 2005).

90 Size distribution of overlapping particles of coarse sands and gravel $(0.7 \mathrm{~mm}$ to $20 \mathrm{~mm}$ ) has also been analysed through statistical image properties (Buscombe \& Masselink, 2009), however determining the sample distribution through regression over the images in a "look-up catalogue" limits the possible target distributions. In summary, these approaches are limited to cases of little or no particle overlap and use samples comprising large aggregates, with further drawbacks including the reliance on a catalogue or segmentation of the image into individual particles.

口 Granulometries (Matheron, 1975) and subsequently pattern spectra (Maragos, 1989), were early morphological operations. They were developed as tools for scale (size) and shape analysis of image content, with initial applications

1 https://lcas.lincoln.ac.uk/wp/research/data-sets-software/ soil-aggregate-size-distribution-dataset/ 
in petrography (i.e. studying the grain structure of rocks). They were used as global (Urbach et al., 2007; Tushabe \& Wilkinson, 2007) as well as patch and region descriptors in general image processing tasks (Chen \& Dougherty, 1994 Bosilj, Aptoula, Lefèvre \& Kijak, 2016). Pattern spectra through opening and closing with reconstruction were used for the granulometric analysis of estuarine and marine sediments (Frančišković-Bilinski, Bilinski, Vdović, Balagurunathan \& Dougherty, 2003), as well as soil section images (Doulamis, Doulamis \& Maragos, 2001) (also including spectra based on area openings). However both methods focus on samples with mostly non-overlapping aggregates.

Pattern spectra based on area openings calculated on a max-tree were used to produce accurate grain size distributions of sands (smallest reported particle size $0.06 \mathrm{~mm}$ ) (Pina, Lira \& Lousada, 2011). Image granulometry was also fonsidered for estimating the size distribution of stone fragments (Salehizadeh \& Sadeghi, 2010). A recent study compared pattern spectra based on different SEs, as well as area openings, closings and their combination, for the assessment of grain size for fine and coarse aggregates of sands and pebbles $(0.125 \mathrm{~mm}$ to $16 \mathrm{~mm}$ ) (Bianconi, Di Maria, Micale, Fernández \& Harvey, 2015) with the best results obtained through attribute morphology. The mean grain size was estimated through regression on the training samples, by assuming a quadratic relation between measured grain size and image granulometry. Image granulometry was also related to the measured mass distribution of the samples. However, this work was validated on prepared samples with predetermined unimodal grain size distribution, while processing partial images of very large samples.

The sample size used in our experiments is several orders of magnitude smaller than the one used previously (Bianconi et al., 2015), and the image acquisition setup produced partially to completely overlapping and touching aggregates. An additional difference is that our sample structure is more complex, resulting in a multimodal distribution of soil aggregates. Finally, while baseline attribute morphology pattern spectra based on min- and max-trees have already been shown to outperform their counterparts with SEs for similar tasks (Bianconi et al. 2015, in this work a more detailed analysis of attribute morphology 
pattern spectra resulting from different image hierarchies was performed.

\section{Methodology}

In this section, we introduce the basic concepts of morphological image proof the system in Fig. 1

\subsection{Morphological processing of binary images}

A 2D binary image $f: E \rightarrow\{0,1\}, E \subseteq \mathbb{Z}^{2}$ is formally defined as an element from a partially ordered set on the image domain. The standard terminology of pixels, connectivity, foreground and background (objects), translation and point-wise maxima and minima operations is used.

A filtering is an image transformation which selectively suppresses image noise, certain image structures or objects. Morphological filters are non-linear and preserve elements based on the geometry and contrast of image pixels and their local neighbourhoods (Soille, 2013). They are characterised by the properties of idempotence (repeated applications of the filter have no effect) and increasingness (preserving the ordering relation between images).

This work relies on a group of filters called openings to measure content. They remove image content, are characterised as anti-extensive and result in images where the pixel values can only be lower or equal to the original. A dual operation adding image content is called a closing and the property extensivity.

The classical openings defined through the interaction of an image with a static SE are now described, as well as the attribute openings belonging to the adaptive morphological operations which are not shape-biased (Breen \& Jones,

155 1996). For a more detailed analysis of openings, closings, and their properties, the reader is referred to (Serra, 1983, Ronse \& Heijmans, 1991).

\subsubsection{Structuring element filtering}

In classical morphology, image transformations can be described as the result of probing the image with a set of a known shape called a structuring element 


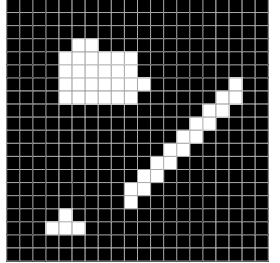

(a) input image

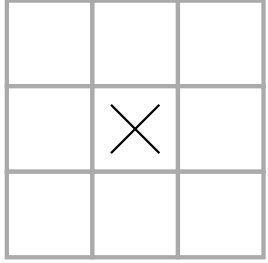

(b) SE

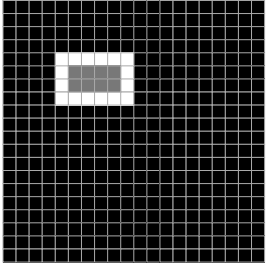

(c) SE opening

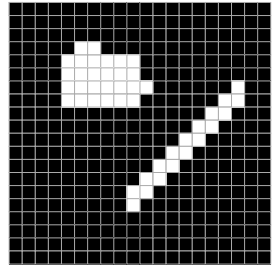

(d) attribute opening for $A(\mathcal{R}) \geq 9$

Figure 2: The opening of an example binary image in (a) with the structuring element (SE) in (b) is shown in (c) with the erosion also indicated by shading. The result of applying an attribute opening using the criterion "area larger than 9" on the same image is shown in (d)

(SE), which is commonly a small symmetric image (e.g. $3 \times 3$ px as shown in Fig. 2(b) chosen based on the prior knowledge about the geometry of the relevant or irrelevant image objects (Soille, 2013).

The basic morphological transformations are erosion and dilation by an SE, equivalent in practice to Minkowski addition and subtraction (Minkowski, 1903 Hadwiger, 1950, Maragos \& Schafer, 1990). Erosion removes image content. Applying an erosion with SE $B$ to an image $f$ results in an image $\epsilon_{B}(f)$ containing all the SE origin locations where the SE is fully covered by foreground pixels in $f$. Dilation is the dual operator of erosion, adding content to the image, and the dilated image $\delta_{B}(f)$ shows the locations of the SE origin where the SE contains at least one foreground pixel in $f$. Erosion (resp. dilation) can be interpreted as assigning to the pixel $p$ in the resulting image the lowest (resp. highest) value of the original image contained in the SE when centred on the pixel $p$.

An SE opening $\Gamma_{B}(f)$ with an SE $B$, used to provide a baseline performance for comparison with the attribute openings implemented through component trees under study in this work (explained in Secs. 3.1.2 and 3.2.2), is defined as an erosion followed by a dilation with the reflected SE. It is sometimes incorrectly defined as an erosion followed by a dilation with the same SE, however this is because reflecting a typical (flat and symmetric) SE does not change it. If the SE fits the image at a certain origin location, all the SE elements are added 
removed as with erosion, but some elements of the preserved foreground regions are partially recovered. The dual closing operation removing small background regions is similarly defined through chaining a dilation followed by an erosion, or can be calculated as an opening of the image complement.

185 In SE morphology, one might use a $3 \times 3 \mathrm{SE}$ such as the one on Fig. 2(b) to remove the image objects smaller than 9 pixels. The resulting image in Fig. 2(c) illustrates the shortcomings of SE morphology: firstly, the elongated object is removed, despite its size, as its shape does not fit the chosen SE, and secondly, the preserved object is not reconstructed to its original shape. This is due to SE morphology being shape-biased (Breen \& Jones, 1996), as opposed to attribute morphology explained in the next section.

\subsubsection{Attribute filtering}

In order to adapt to the image content, attribute filters work directly on the foreground regions of the image based on a given connectivity relation (here, the standard 4-connectivity is used), formally called the connected components, $C C(f)$, of the image, $f$. They belong to the family of connected operators which work directly on $C C(f)$ (Heijmans, 1999) and coarsen the image partition. The set $C C(f)$ consists of connected foreground components of maximal extent, and a single connected component is denoted by $C C(f)^{i}$ (with $i$ from some index set).

To make decisions about the connected components of the image, we can evaluate different criteria for each $C C(f)^{i}$. A criterion $\mathcal{K}$ operating on sets is said to be increasing if, when the criterion holds for a set $X$, it also holds on all the supersets of $X$. A common way to define a criterion $\mathcal{K}$ is through comparing the value of an attribute $A(\cdot)$ calculated on a region $X$ to a threshold $T$, where using an increasing attribute such as area results in an increasing criterion $\mathcal{K}_{A, T}$. We say that $X$ satisfies $\mathcal{K}_{A, T}$ if and only if $A(X) \geq T$. Given an increasing criterion $\mathcal{K}$, the trivial opening $($ Serra \& Vincent, 1992$) \Gamma_{\mathcal{K}}(X)$ of a connected 
set preserves the set $X$ if it satisfies the criterion $\mathcal{K}$. This definition is extended to images by applying the opening to all the connected components $C C(f)$ :

$$
\Gamma_{\mathcal{K}}(f)=\bigcup_{i} \Gamma_{\mathcal{K}}\left(C C(f)^{i}\right)
$$

While a number of increasing attributes can be used to filter image components according to their size (Breen \& Jones, 1996), in this work we focus on the area opening (Vincent, 1993a), where the threshold choices correspond to the mesh sizes on the physical sieves used in manual soil size distribution analysis.

\subsection{Extension to greyscale images}

To be applicable as image analysis tools, morphological methods and techniques need to be extended to greyscale images. A 2D greyscale image (with fixed-precision pixel values) is formally defined as $\mathbf{f}: E \rightarrow\left\{0,1, \cdots, t_{\max }\right\}, E \subseteq$ $\mathbb{Z}^{2}$. The core principles behind defining greyscale morphology are threshold decomposition and superposition (Serra, 1983 , Maragos \& Ziff, 1990). Efficient implementations of different filters are achieved by relying on component trees defining hierarchies of connected components (Bosilj, Kijak \& Lefèvre, 2018).

\subsubsection{Threshold decomposition and superposition}

A greyscale image $\mathbf{f}$ can be decomposed into its cross-sections or upper level sets (Monasse \& Guichard, 2000). The upper level set $L^{k}(\mathbf{f})$ of the image $\mathbf{f}$ at the level $k$ contains all the pixels $\mathbf{f}(p)$ with values higher than $k, L^{k}=$ $\{p \in \mathbf{f} \mid \mathbf{f}(p) \geq k\}$. These sets are nested and follow an inclusion relationship, $L^{0} \subseteq L^{1} \cdots \subseteq L^{t_{\max }}$. Similarly, the lower level sets $L_{k}$ contain all the image pixels lower than a value threshold. The value of the image $\mathbf{f}$ at a pixel $p$ can be obtained as the largest threshold value $k$ for which $p$ is included in the associated upper level set $L^{k}$ :

$$
\mathbf{f}(x)=\sum_{k=1}^{t_{\max }}\left[L^{k}(\mathbf{f})\right](p)=\max \left\{k \mid p \in L^{k}(\mathbf{f})\right\} .
$$

Such representation of a greyscale image as the sum of its successive upper level sets is referred to as the threshold decomposition or threshold superposition principle (Serra, 1983, Maragos \& Ziff, 1990). 
This offers a template for extending the increasing binary filters to greyscale (Serra, 1983, Vincent, 1993b) by applying the filter to all upper level sets and summing the results:

$$
[\psi(\mathbf{f})](x)=\sum_{k=1}^{t_{\max }}\left\{\psi\left[\left(L^{k}(\mathbf{f})\right]\right\}(p)\right.
$$

The greyscale filters which can be expressed as Eq. (3) are called flat operators (Wendt, Coyle \& Gallagher, 1986) and keep a number of properties of their bi-

220

Breen \& Jones, 1996). While Eq. (2) provides a theoretical basis for extending binary to greyscale morphology, applying the transformation to every level set results in a very slow implementation (Vincent, 1993b). Instead, greyscale attribute morphology relies on the component trees discussed hereafter, and SE age partitions as cross-sections, which are typically extrema-oriented are distinguished from partitioning hierarchies (see Fig. 4) with nested image partitions as cross-sections, which are better at representing regions at intermediate values (Bosilj et al. 2018).

240 . The min and max-trees are seminal morphological hierarchies (Breen \& Jones, 1996, Salembier et al., 1998), modelling the inclusion relations between the upper and lower level sets of the image which are nested. These are dual 


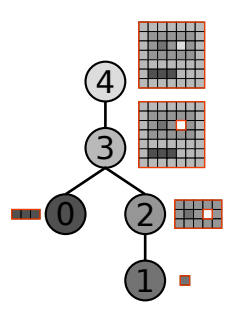

(a)

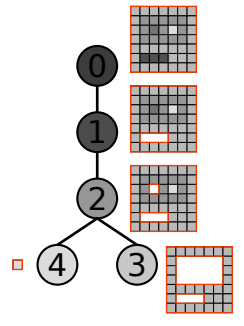

(b)

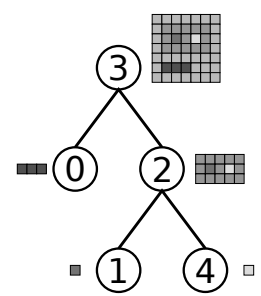

(c)

\begin{tabular}{|l|l|l|l|l|l|l|}
\hline 3 & 3 & 3 & 3 & 3 & 3 & 3 \\
\hline 3 & 2 & 2 & 2 & 2 & 2 & 3 \\
\hline 3 & 2 & 1 & 2 & 4 & 2 & 3 \\
\hline 3 & 2 & 2 & 2 & 2 & 2 & 3 \\
\hline 3 & 3 & 3 & 3 & 3 & 3 & 3 \\
\hline 3 & 0 & 0 & 0 & 3 & 3 & 3 \\
\hline 3 & 3 & 3 & 3 & 3 & 3 & 3 \\
\hline
\end{tabular}

(d)

Figure 3: The three different inclusion trees of a toy image (d) The min-tree is displayed in (a) while its dual max-tree is shown in (b) The self-dual tree of shapes is shown in (c) The (grey) levels of the nodes are displayed in the nodes, and the corresponding regions are shown beside the nodes.

hierarchies belonging to the class of inclusion trees, and are well-suited for representing dark and bright image components, respectively. Examples are shown in Fig. 3(a) and (b)

The tree of shapes (ToS) (Monasse \& Guichard, 2000) unifies the representation of bright and dark image structures, producing a single self-dual image representation, treating bright and dark components equally based on their absolute contrast with their background. It comprises all the connected components of both upper and lower level sets with their holes filled, which also form an inclusion hierarchy. An example of a ToS is shown in Fig. 3(c),

The $\alpha$-tree is a partitioning tree based on the local range of its components (Soille, 2007, 2008) (also sometimes referred to as quasi-flat zone hierarchy (Cousty, Najman, Kenmochi \& Guimarães, 2018)). The finest segmentation contained in the leaves of the tree comprises connected components of maximum extent of pixels at the same grey level, which are then merged according to the local neighbour similarity. As such, this hierarchy is capable of representing both bright, dark and intermediate level regions. However, due to the locality of the criterion used, the grey level variations within regions tend to be much higher than $\alpha$ when the grey levels in the image increase and decrease gradually, called the chaining effect (Soille, 2008). An example of the hierarchy is shown 


\begin{tabular}{|l|l|l|l|l|}
\hline 0 & 0 & 3 & 3 & 5 \\
\hline 1 & 2 & 4 & 3 & 5 \\
\hline 4 & 1 & 2 & 5 & 2 \\
\hline 4 & 5 & 2 & 0 & 3 \\
\hline 2 & 3 & 0 & 1 & 0 \\
\hline
\end{tabular}

(a)

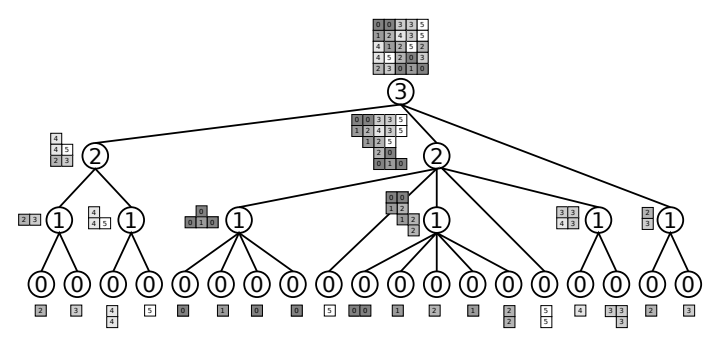

(b)

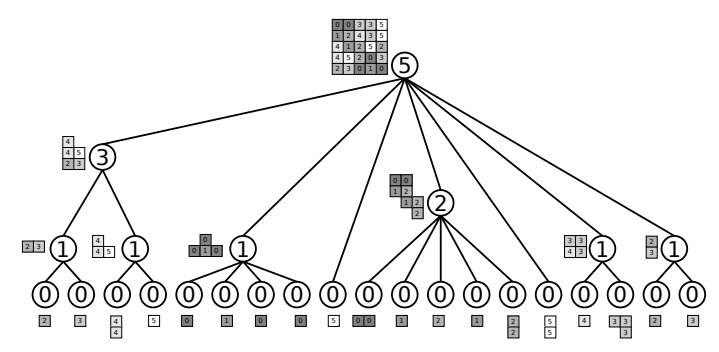

(c)

Figure 4: For the toy image in (a) the $\alpha$-tree is displayed in (b) while the constrained hierarchy $(\omega)$-tree is shown in (c) The $\alpha$ (resp. $(\omega)$ ) levels are displayed in the nodes and indicated by their height, with regions displayed besides the nodes.

in Fig. 4(b), with the chaining effect observable for $\alpha=2$.

The most notable constrained connectivity hierarchy designed to deal with the chaining effect is the $(\omega)$-tree (Soille, 2008), which rearranges the regions of the $\alpha$-tree according to their global intensity range, removing some of the regions but providing better grouping per level than just a local measure (see Fig 4(c).

Attribute filtering is then implemented through evaluating the attribute value on every region present in the hierarchy and discarding those not satisfying the criterion, followed by reconstituting the image from a filtered tree. Due to the increasing property of the opening operations and the nested nature of the regions ordered into a hierarchy, a complete traversal of the tree can be avoided. According to the direct filtering rule (Salembier \& Wilkinson, 2009) during the top-down traversal, if a parent node does not satisfy a given criterion, 

for calculating the cumulative distribution of image content according to size, and can be interpreted as consecutively sieving the image with an increasing mesh size. It has been extended to shape granulometries (Urbach \& Wilkinson 2002 used for characterising the distribution of image component shapes and

Size granulometries are implemented through applying a series of openings with increasing size $\left\{\Gamma_{t_{i}}\right\}, t_{i+1}>t_{i}$, where every consecutive opening removes more detail from the image. A size granulometry of an image $\mathbf{f}$ is denoted as 
$\left\{g_{\Gamma, t_{i}}(\mathbf{f})\right\}$, and calculated as the amount of detail remaining in the image after each filtering operation:

$$
g_{\Gamma, t_{i}}(\mathbf{f})=\mathcal{M}\left[\Gamma_{t_{i}}(\mathbf{f})\right],
$$

where $\mathcal{M}$ is a measure of image content. As image openings interact with the image components brighter than the background, a granulometry by opening only contains information about the size distribution of foreground image elements. To study the size distribution of the background components, the image can be filtered with a series of closings of increasing size $\left\{\Phi_{t_{i}}\right\}, t_{i+1}>t_{i}$. Whilst the term anti-granulometry is sometimes used (Soille, 2013), as this operation is not based on an increasing operation, it will be referred to simply as granulometry by closing through the paper, as the suitability of both approaches for estimation of soil aggregate size distribution is studied.

\subsubsection{Pattern spectra}

Unlike granulometries, which note the amount of remaining image content, pattern spectra measure the amount of image detail removed between two consecutive filtering operations. A size pattern spectrum $\left\{s_{\Gamma, t_{i}}(\mathbf{f})\right\}$ is obtained from a granulometry $\left\{\Gamma_{t_{i}}\right\}$ by storing the differences in measures of the two successive filtered images:

$$
\begin{aligned}
s_{\Gamma, t_{i}}(\mathbf{f}) & =\mathcal{M}\left[\Gamma_{t_{i-1}}(\mathbf{f})\right]-\mathcal{M}\left[\Gamma_{t_{i}}(\mathbf{f})\right] \\
& =g_{\Gamma, t_{i-1}}(\mathbf{f})-g_{\Gamma, t_{i}}(\mathbf{f}) \\
s_{\Gamma, t_{\min }}(\mathbf{f}) & =\mathcal{M}(\mathbf{f})-\mathcal{M}\left[\Gamma_{t_{\min }}(\mathbf{f})\right] \\
& =\mathcal{M}(\mathbf{f})-g_{\Gamma, t_{\min }}(\mathbf{f}) .
\end{aligned}
$$

In the early research on pattern spectra, these values were sometimes normalised with a scale parameter $t_{i}$ (Maragos, 1989). This is because the pattern spectrum can be interpreted as a probability density function (Maragos, 1989 Dougherty, Pelz, Sand \& Lent, 1992) in its simplest form, which is a histogram lative distribution function. 
Efficient implementations of both granulometries and pattern spectra rely on attribute openings implemented through component trees. The attribute of interest is calculated for all the regions during tree construction, followed by determining the first opening from the sequence $\left\{\Gamma_{t_{i}}\right\}$ interacting with each region and the bin $i$ to which the region will contribute. This approach requires only a single traversal of the tree to calculate the whole $\left\{g_{\Gamma, t_{i}}(\mathbf{f})\right\}$ and $\left\{s_{\Gamma, t_{i}}(\mathbf{f})\right\}$, relying on the fact that the applied filters are increasingly coarser and any content removed by $\Gamma_{t_{i}}$ will also be removed by $\Gamma_{t_{j}}$ for all $i<j$.

\subsubsection{Content measures}

Finally, the content measures used in Eqs. (4) and (5) are defined. The most commonly used measure is the Lebesgue measure, corresponding to the image volume (with pixel values heights) for 2D images used in this paper:

$$
\mathcal{M}_{\mathrm{vol}}(\mathbf{f})=\sum_{k=0}^{t_{\max }} A\left[L^{k}(\mathbf{f})\right]=\sum_{k=0}^{t_{\max }} \sum_{i} A\left\{C C\left[L^{k}(\mathbf{f})\right]^{i}\right\}
$$

As the max-tree is a hierarchical representation of the upper level sets of the image, calculating this measure corresponds to summing up the areas of regions present in the tree, weighted by their contrast with their parent. The need to weight the region areas in the calculation comes from the fact that the max-tree encodes only the first threshold value for which each region appears in the tree.

However, since the ToS can simultaneously remove both bright and dark image components, it is possible that $\mathcal{M}_{\mathrm{vol}}(\mathbf{f})=\mathcal{M}_{\mathrm{vol}}[\Gamma(\mathbf{f})]$ are equal despite $\mathbf{f} \neq \Gamma(\mathbf{f})$. Therefore, we propose a different measure, which we name dynamic volume, to avoid this undesirable behaviour on the tree of shapes. Every tree can be represented as a set of regions $\mathcal{H}=\left\{D_{\mathcal{H}}^{i}\right\}$ with $i$ from some index set (e.g. the connected components of the upper level sets for the max-tree) (Bosilj et al. 2018). The grey level of the region $D_{\mathcal{H}}^{i}$ is denoted by $G\left(D_{\mathcal{H}}^{i}\right)$ where for the inclusion trees this is set to the grey level of new pixels added in each node, and for the partitioning trees to the average of all grey level values in the region. The term $P\left(D_{\mathcal{H}}^{i}\right)$ denotes a parent of the region $D_{\mathcal{H}}^{i}$, which is the smallest region 
containing $D_{\mathcal{H}}^{i}: P\left(D_{\mathcal{H}, i}\right)=D_{\mathcal{H}}^{j} \in \mathcal{H}$ such that $D_{\mathcal{H}}^{i} \subset D_{\mathcal{H}}^{j}$ and $\nexists D_{\mathcal{H}}^{k}$ such that $D_{\mathcal{H}}^{i} \subset D_{\mathcal{H}}^{k} \subset D_{\mathcal{H}}^{j}$.

Using these definitions, the dynamic volume $\mathcal{M}_{\text {dvol }}$ can be defined as:

$$
\mathcal{M}_{\mathrm{dvol}}\left(\mathbf{f}_{\mathcal{H}}\right)=\sum_{i} A\left(D_{\mathcal{H}}^{i}\right) \times\left|G\left(D_{\mathcal{H}}^{i}\right)-G\left[P\left(D_{\mathcal{H}}^{i}\right)\right]\right|
$$

where $\mathbf{f}_{\mathcal{H}}$ denotes an image $\mathbf{f}$ represented by a hierarchy $\mathcal{H}$. Note that for the min and max-tree hierarchy, $\mathcal{M}_{\mathrm{vol}}\left(\mathbf{f}_{\mathcal{H}}\right)=\mathcal{M}_{\mathrm{dvol}}\left(\mathbf{f}_{\mathcal{H}}\right)$.

The region count is also used as a content measure, which is topologically invariant to both the spatial extent and the greylevel variations induced by the filtering (Cavallaro, Falco, Dalla Mura \& Benediktsson, 2017). The measure was originally defined to measure the number of connected components affected by the filtering. However, since this work defines content measures as descriptors of single images, as opposed to Cavallaro et al. (2017) who define it on a pair consisting of an original and a filtered image, the region count is redefined as $\mathcal{M}_{\text {count }}\left(\mathbf{f}_{\mathcal{H}}\right)$ to reflect the number of connected components in the image or the hierarchy:

$$
\mathcal{M}_{\text {count }}\left(\mathbf{f}_{\mathcal{H}}\right)=\left|D_{\mathcal{H}}\right| \text {. }
$$

${ }_{345}$ When the proposed definition of $\mathcal{M}_{\text {count }}$ is used with Eq. (5) to define a value of the pattern spectrum, obtained as the difference of content measures between two consecutive filtered images, the obtained values are equal to those obtained through the original definition (Cavallaro et al., 2017). The third measure under consideration in the literature (Cavallaro et al., 2017) measures the number of pixel values changed by a filtering. However, this is not a direct measure of image content, but rather a difference measure between two images, calculated from the filtered image paired with the original. As such, it is not suitable for use in Eqs. (4) and (5) and was therefore omitted from this study.

Pattern spectra, as described in this section, are at the core of the proposed digital sieving method. We calculate $\left\{s_{\Gamma, t_{i}}(\mathbf{f})\right\}$ for all the soil sample images, according to Eq. (5) and using both content measures from Eqs. (7) and (8). The pattern spectrum is then presented to the trained regression model, which 
maps the image component size distribution, expressed in terms of pixels and measured through Eqs. (7) and (8), into a soil aggregate size distribution in terms of aggregate dimension measured through aggregate weight or volume.

\section{Dataset Description}

In this section, we describe the dataset collected to study the application of image processing techniques to soil structure assessment. Soil samples were collected from arable soils using a spade to a depth of $200 \mathrm{~mm}$, a method similar 
Table 1: Visual appearance of the collected soil samples A - D at the far scale (area $250 \mathrm{~mm} \times 250 \mathrm{~mm})$.

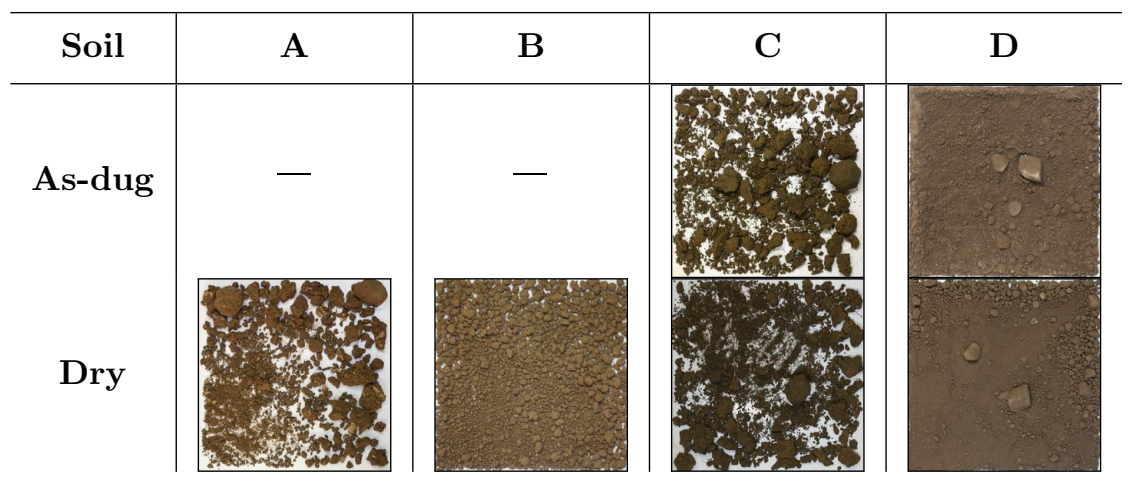

To create a uniform moisture content across samples, all samples were dried in an oven at $60^{\circ} \mathrm{C}$ for 24 hours, a standard procedure in soil science. In addition, we collected images of the soils $\mathrm{C}$ and $\mathrm{D}$ as-dug, i.e. before drying them in the oven. The dried samples were then sieved gently by hand through a stack of 7 sieves of mesh sizes $0.212,2,3.15,5,9.5,25$ and $50 \mathrm{~mm}$ diameter, a similar approach to laboratory aggregate methods (Le Bissonnais, 1996). To measure the aggregate size distribution, we examine the remaining soil fraction on each of the sieves. The soil fraction is first weighted on scales, followed by measuring the volume by water displacement. The aggregate size distribution of samples A-D is shown in Table 2 ,

Square surfaces of three different sizes (see Table 3) were drawn on a white tray (shown in Fig. 5(a), then the soils were placed in the tray and manipulated with brushes to fit the marked surface. This setup allowed us to collect images at different pixel resolutions, as well as examine the influence of the visible background surface in the sample images. Two sets of images were produced, using a professional and an everyday camera, therefore obtaining images of different quality. The first set of images was taken with a Canon EOS 40D camera, which was placed at a fixed height to provide a top-down view of the samples and manually focused. This produced images of size $3888 \mathrm{px} \times 2592 \mathrm{px}$, 
Table 2: The aggregate size distribution of the samples used as ground truth. All values are expressed in [\%].

\begin{tabular}{c|c||c|c|c|c|c|c|c}
\hline \multicolumn{2}{c|}{ mesh size [mm] } & 0.212 & 2 & 3.15 & 5.0 & 9.5 & 25 & 50 \\
\hline \hline \multirow{5}{*}{ weight } & A & 0.73 & 2.65 & 1.73 & 4.26 & 14.94 & 42.06 & 33.63 \\
& B & 1.10 & 7.99 & 8.89 & 16.34 & 47.65 & 18.03 & 0.00 \\
& C & 0.53 & 5.64 & 3.96 & 6.52 & 15.93 & 49.98 & 17.44 \\
& D & 8.85 & 48.06 & 8.15 & 9.07 & 11.45 & 7.66 & 6.76 \\
\hline \multirow{5}{*}{ volume } & A & 0.54 & 2.69 & 2.69 & 5.37 & 16.13 & 40.32 & 32.26 \\
& B & 1.09 & 8.70 & 9.78 & 16.30 & 46.20 & 17.93 & 0.00 \\
& C & 0.66 & 5.30 & 3.97 & 8.61 & 16.56 & 47.68 & 17.22 \\
& D & 8.23 & 50.63 & 7.60 & 8.86 & 11.39 & 6.96 & 6.33
\end{tabular}

see Fig. 5(b), The fixed height was determined empirically to allow for maximal pixel resolution for each of the three marked surface sizes (see Table 3 for details). The second set of images was taken with a phone camera (iPhone 6), which was held as close as possible to the tray so that the whole sample was captured, but without a fixed height. This produced images of smaller size of $3264 \mathrm{px} \times 2448 \mathrm{px}$, not perfectly focused and more sensitive to lighting, which more closely reflects the target in-field applications.

Finally, after taking the images, the corners of the marked square were taken as markers for applying a homography to the images to produce a top-down 415 image, as well as for discarding the parts of the image not containing the sample. The size of the resulting images (example in Fig. 5(c) was chosen close to the original resolution along the shorter image axis, in order to minimise the rescaling effects. The resulting resolution is $2500 \mathrm{px} \times 2500 \mathrm{px}$ for the images taken with the professional camera, and $2000 \mathrm{px} \times 2000 \mathrm{px}$ for the images taken with the phone. The soils were fitted into each of the marked surfaces twice, resulting in two different arrangements of each soil at each scale for a total of 36 image pairs, taken by the digital camera and smartphone, in the dataset. 
Table 3: The different scale settings used.

\begin{tabular}{c||c|c|c}
\hline Scale & close & middle & far \\
\hline \hline Area $[\mathrm{cm} \times \mathrm{cm}]$ & $15 \times 15$ & $20 \times 20$ & $25 \times 25$ \\
Camera height $[\mathrm{cm}]$ & 60 & 78 & 94 \\
Resolution (camera) $\left[\mathrm{px} \mathrm{mm}^{-1}\right]$ & 16.7 & 12.5 & 10 \\
Resolution (phone) $\left[\mathrm{px} \mathrm{mm}^{-1}\right]$ & 13.3 & 10 & 8
\end{tabular}

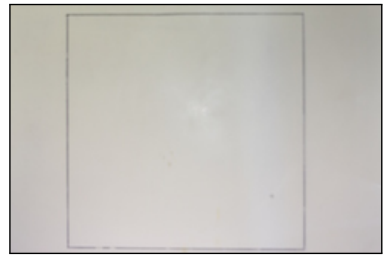

(a)

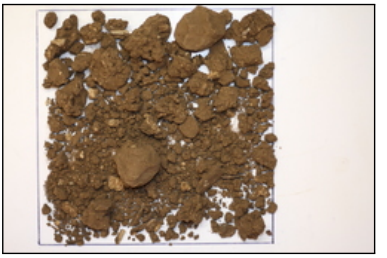

(b)

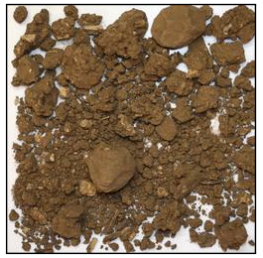

(c)

Figure 5: The image acquisition setup: (a) the middle $(200 \mathrm{~mm} \times 200 \mathrm{~mm})$ square marked on the tray, (b) one of the original images of soil $\mathrm{A},(\mathrm{c})$ the final image obtained by applying the rectifying homography.

\section{Experimental setup}

This section presents the experiments designed to examine the ability to pre-

dict the soil size distribution from image pattern spectra based on different image hierarchies from attribute morphology, as well as SE morphology, and assess their potential for developing an imaging pipeline for performing quantitative soil analysis. All images have been loaded as greyscale for further processing, relying on the internal conventions of the libjpg codec for the conversion from colour images.

The bin thresholds were chosen in two different ways and the performance of the resulting descriptors compared. The upper limit for the largest bin was set to the largest expected particle size of $50 \mathrm{~mm}$ (all the aggregates of all the samples A-D passed through a $50 \mathrm{~mm} \times 50 \mathrm{~mm}$ sieve). We firstly tested logarithmic

${ }_{335}$ binning, which is commonly used with pattern spectra descriptors Bosilj et al. 
2016). Secondly, the physical dimensions of each sieve mesh were used to calculate the area of grid openings in pixels for each of the scales, and used those as bin limits. The number of bins in the logarithmic binning was set to the same number of bins determined by the physical sieves used, $b=7$.

We used three different inclusion trees (min and max-tree, ToS) and two different partitioning trees ( $\alpha$ and $(\omega)$-tree) for pattern spectra calculation (outlined in Sec. 3.2.2), as well as the sum of the histograms obtained from the minand max-trees (the corresponding bins were summed to obtain a new histogram of the same length, denoted as $\min +\max )$. Each image was described by its associated pattern spectrum, normalised so that the sum of all histogram values equals 1. As a baseline, we also used the pattern spectra obtained from a granulometry through SE opening, as well as an anti-granulometry through SE closing. The approaches used to calculate the different pattern spectra are summarised in Table 4 .

The pattern spectra were then used to train a regression model, which was evaluated using leave-one-out validation (with a single pair of camera and phone images held out from training in each iteration). Several regression models were evaluated, and the best performance obtained with regression based on Gaussian Processes (Williams \& Rasmussen, 2006), stochastic processes specified by their mean and covariance functions. It was empirically determined that using the absolute exponential kernel led to the best performance. The length scale parameter controls the variability of the learned function and reflects the confidence in the training data. The best kernel parameter length scale $=0.1$ was found by a search through the parameter space.

To measure the quality of the regression outputs, the Wasserstein distance (also known as earth mover's distance) (Villani, 2008) between the manually measured aggregate distributions and predicted distributions was calculated. The metric originates from optimal transport theory (Bonneel, Peyré \& Cuturi 2016), and is based on interpreting probability histograms as heaps of sand or dirt at certain locations and considering the most efficient way to reshape one histogram into another in terms of the distance the dirt has to be moved 
Table 4: The different approaches used to calculate pattern spectra.

\begin{tabular}{c||c}
\hline Abbreviation & Description \\
\hline \hline opening & granulometry based on SE opening \\
closing & granulometry based on SE closing \\
$\alpha$ & tree-based granulometry from the $\alpha$-tree \\
$(\omega)$ & tree-based granulometry from the $(\omega)$-tree \\
$\min$ & tree-based granulometry from the min-tree \\
$\max$ & tree-based granulometry from the max-tree \\
$\min +\max$ & sum of tree-based granulometries from the min and max-tree \\
ToS & tree-based granulometry from the tree of shapes
\end{tabular}

to achieve this. Histograms with little overlap but differing only by a small displacement (corresponding to slightly overestimating or underestimating the aggregate size) will still be considered as similar. In the concrete case of aggregate size distribution of soil samples, the weight or volume of soil in each bin corresponds to the amount of dirt in a heap, while the mesh size which dictates the diameter of aggregates determines the location of that heap. As all of our histograms were normalised to unit weight, the metric describes the average error in the estimated aggregate diameter.

\section{Results and Discussion}

As the error distributions are heavily tailed, we have chosen to express our results in terms of median and median absolute deviation as $\tilde{e} \pm M A D$, where $\tilde{e}=\operatorname{median}(e)$ and $\mathrm{MAD}=\operatorname{median}\left(\left|\left(e_{i}-\tilde{e}\right)\right|\right)$. The results indicate that pattern spectra descriptors show promising ability in predicting the soil size distribution, with the best predictor resulting in the expected error in aggregate diameter of $(1.1 \pm 1.0) \mathrm{mm}$ when measuring both in terms of weight (Fig. 6) and volume (Fig. 7).

Such results suggest that the potential for pattern spectra descriptors to estimate soil size distributions are more robust on the "bigger" structured soils, 


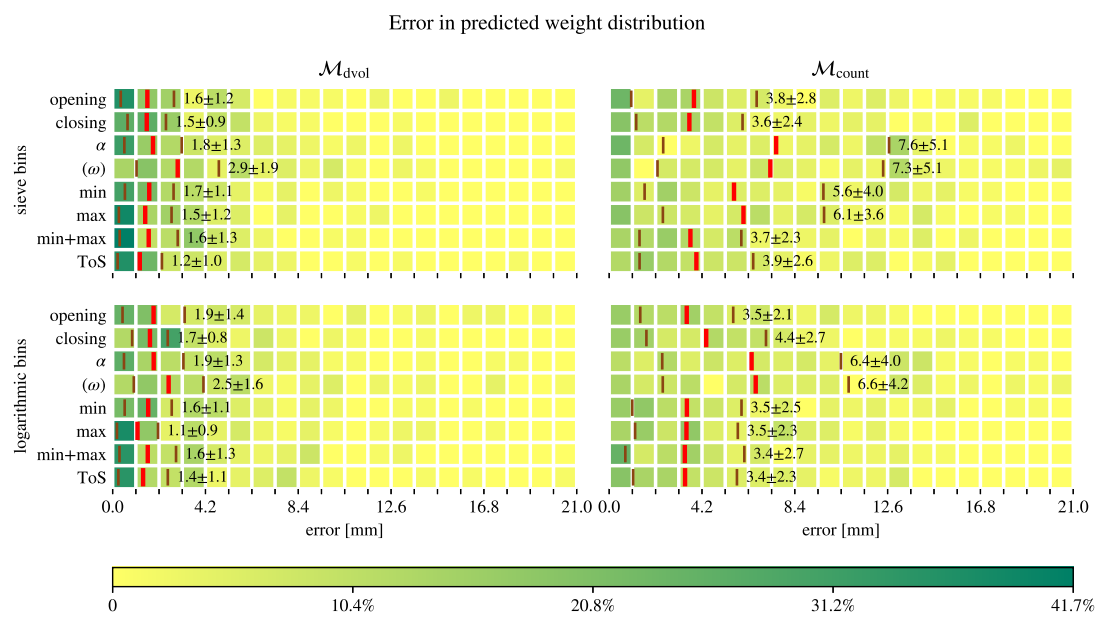

Figure 6: Distribution of errors for regression systems predicting the size distribution of the samples, based on weight from pattern spectra derived from different hierarchies and classical granulometries, using both content measures and binning strategies. $\tilde{e} \pm M A D$ for each approach is shown on top of the corresponding error distribution.

those that consist of larger cloddy structures or are made up of larger macroaggregates (2 to $8 \mathrm{~mm}$ ) (Márquez, Garcia, Cambardella, Schultz \& Isenhart 2004). Such structures are more consistent with the scale of in-field assessments, where a block of soil would been broken down into clods or aggregates (Shepherd 2009, Ball et al., 2007) displaying a structural range from a few millimetres (aggregates) up to many centimetres wide (cloddy structures). The results, however, display more potential for error when assessing size distributions at the smaller scale, such as those expected in laboratory analysis where assessments typically aim at differentiating between smaller macro aggregates (0.25 to $2 \mathrm{~mm}$ ) and micro aggregates (up to $0.25 \mathrm{~mm}$ ) (Márquez et al., 2004).

The best results were obtained using the $\mathcal{M}_{\mathrm{dvol}}$ measure, where the expected error for all the approaches is less than $3 \mathrm{~mm}$, while the $\mathcal{M}_{\text {count }}$ measure seems to be a less accurate predictor, reaching expected errors larger than $7 \mathrm{~mm}$, which agrees with the findings of the experiments on the classification problem Bosilj et al. 2019). However, contrary to the classification study (Bosilj et al. 2019), 


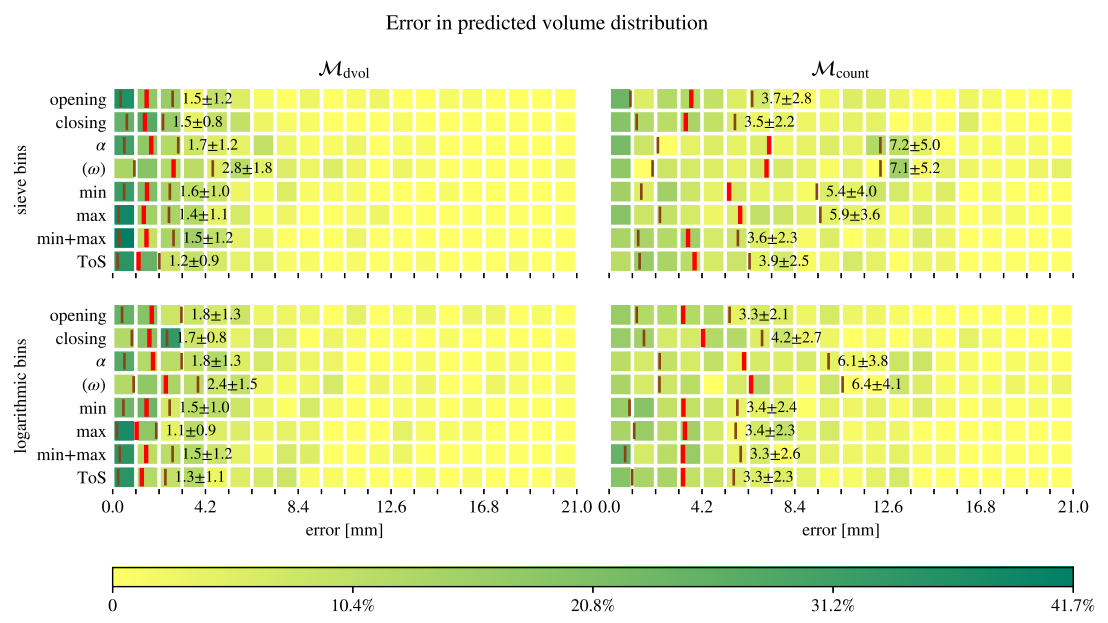

Figure 7: Distribution of errors for regression systems predicting the size distribution of the samples, based on volume from pattern spectra derived from different hierarchies and classical granulometries, using both content measures and binning strategies. $\tilde{e} \pm M A D$ for each approach is shown on top of the corresponding error distribution.

we found that inclusion trees are much better than partitioning trees for predicting both weight and volume distributions. We also found that the predictions produced using logarithmic binning, which does not require knowledge of all the bin limits in advance, are very similar in quality to those using the mesh sizes as bin limits. While the best results were achieved using the max-tree (which is the attribute counterpart to SE opening) (Salembier et al. 1998), logarithmic binning and the $\mathcal{M}_{\mathrm{dvol}}$ measure, we found that it does not provide a consistent improvement over SE opening, as indicated in the previous study comparing classical and attribute morphology for predicting aggregate size distribution (Bianconi et al., 2015). The tree of shapes (Monasse \& Guichard, 2000) provides the most stable results of good quality across all parameter combinations, achieving the best expected error in aggregate diameter of $(1.2 \pm 1.0) \mathrm{mm}$ when relying on mesh sizes as bin limits and the $\mathcal{M}_{\text {dvol }}$ measure.

For the best predictor, we also show the cumulative size distributions for each of the samples in Fig. 8, as well as examples of single predictions that are 


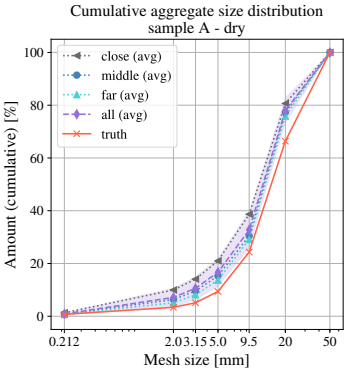

(a)

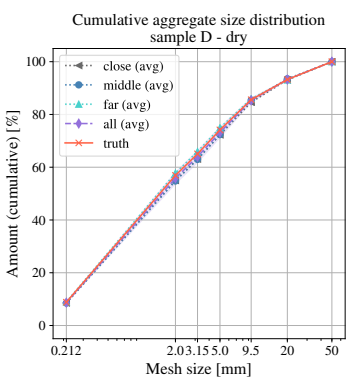

(d)

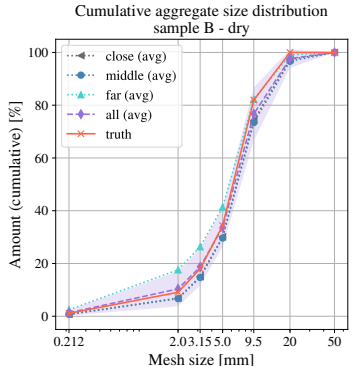

(b)

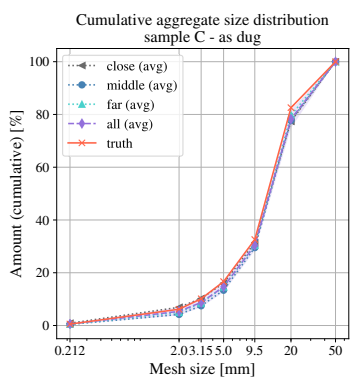

(e)

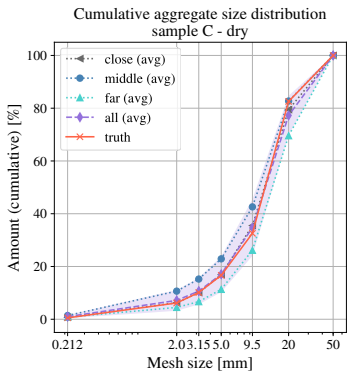

(c)

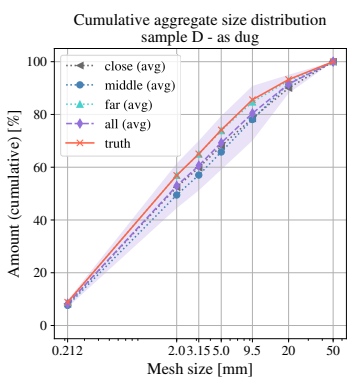

(f)

Figure 8: Cumulative size distributions for samples A - D (dry and as-dug) are shown in (a) (f) For each sample, we separately show the average prediction for each of the three scales (labelled as 'close', 'middle' and 'far'), averaged from 4 predictions based on images of 2 different configurations taken by camera and phone (where the amounts per bin were averaged after calculating the cumulative distribution). We also show the average of all 12 predictions per sample (labelled as 'all'), with shading representing the standard deviation. The manually measured size distributions are labelled as 'truth'.

weight, as the quality of the results is similar to predicting distribution in terms of volume. We can observe that the most difficult size distribution to predict was that of sample A (where the actual cumulative distribution does not lie within one standard deviation of the average prediction), while the best predictions were obtained for samples $\mathrm{C}$ and D. However, we can still clearly distinguish between all the soil samples based on any of the predicted distributions. We can also see that drying the sample does not always lead to a better prediction 


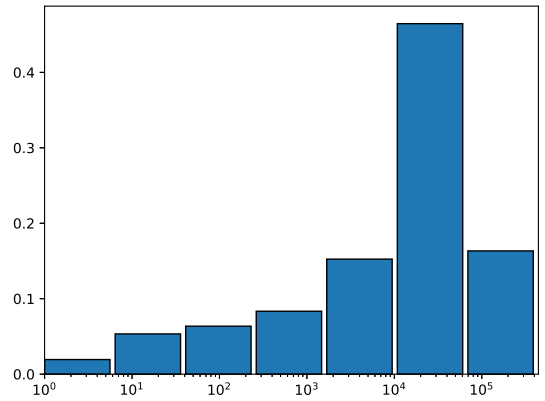

(a)

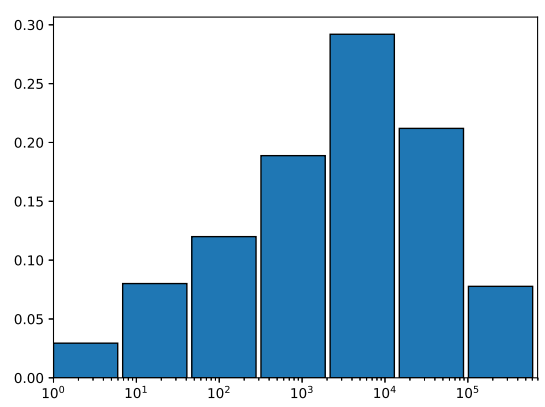

(c)

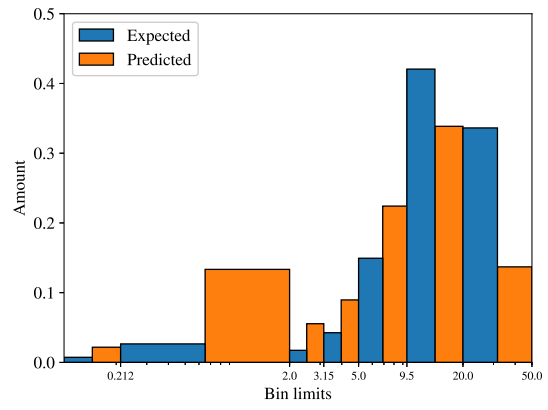

(b)

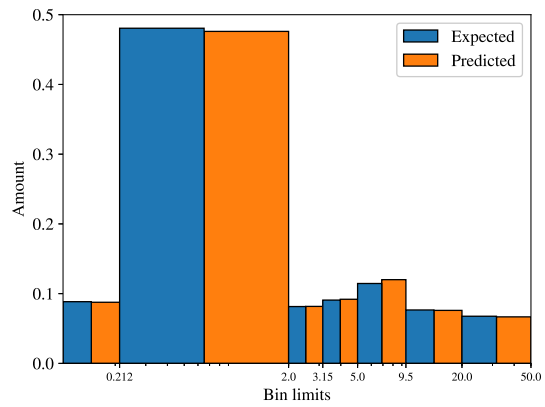

(d)

Figure 9: Histogram and prediction of samples with worst and best classification score for the best classifier in terms of sample weight (max-tree, logarithmic binning, $\mathcal{M}_{\mathrm{dvol}}$ measure): worst $(e=10.72 \mathrm{~mm}$ ) for sample A at close scale (phone) in (a) and (b) and best $(e=$ $0.08 \mathrm{~mm}$ ) for sample D (dry) at close scale (camera) in (c) and (d)

(compare Figs. 8(e) and 8(c)). This shows further potential for the method to be adopted for field-scale "as dug" soil structure assessment, where the soil sample is not subject to any drying preparation. The best predictions such as the one shown in Fig. 9(d) have the size class contributions predicted up to the percentage precision. While it can be observed that the worst prediction in the dataset, shown in Fig. 9(b), has a tendency to under-estimate the predicted size of the particles, the final result still contains useful information as the dominant size class in the aggregate was predicted correctly. We also found no significant difference in performance between the camera and phone images. While these 
are excellent results, we plan to further investigate the performance on a larger number of samples, to exclude the possibility of overfitting to a small dataset.

To illustrate the performance of the best predictor, the cumulative size distributions for each of the samples in Fig. 8 as well as examples of single predictions that are most and least similar to the target output are shown in Fig. 9 These are shown in terms of mass, as the quality of the results is similar to predicting distribution in terms of volume. It can be seen that the most difficult size distribution to predict was that of sample A (where the measured cumulative distribution does not lie within one standard deviation of the average prediction), whilst the best predictions were obtained for samples $\mathrm{C}$ and D. However, all the soil samples can be distinguished between based on any of the predicted distributions. It can also be seen that drying the sample does not always lead to a better prediction (comparing Figs. 8(e) and 8(c) . This shows further potential for the method to be adopted for field-scale "as dug" soil structure assessment, where the soil sample is not subject to any drying preparation. The best predictions, such as the one shown in Fig. 9(d), had size class contributions predicted up to the percentage precision. While it can be observed that the worst prediction in the dataset, shown in Fig. 9(b), had a tendency to under-estimate the predicted size of the particles, the final result still contained useful information since the dominant size class in the aggregate was predicted correctly. Also no significant difference was found in performance between the digital camera and smartphone images. While these are excellent results, it is planned to further investigate performance using a larger number of samples, to exclude the possibility of overfitting caused by a small dataset.

\section{Conclusions and Future Work}

The suitability of pattern spectra for determining the soil aggregate size distribution from soil sample images has been confirmed by including more soil sample images and directly predicting the soil aggregate size distribution measured both in terms of mass and volume. Our experiments were designed to 
work with small sample sizes, and examined the performance of the descriptors under scale changes and the presence of visible background. The suitability of different component trees for soil structure analysis was studied and the results compared to the findings of a previous study on soil re-identification Bosilj

565 et al. 2019). The best performance was achieved using the max-tree, while the ToS achieved consistent good performance across all parameter combinations. Logarithmic binning, which does not require knowledge of all sieve mesh dimensions before descriptor calculation, was found not to be detrimental towards performance, which could remove the necessity of detecting a reference frame to indicate set size in the sample images. The method also performed well on images of soil samples which did not undergo any drying preparation.

Error assessments indicate that the method has potential to be adopted for analysing soil samples displaying larger structures, ranging from millimetres up to several centimetres, as typically found in "as dug" samples, rather than looking at smaller aggregates (micro-aggregates). As part of future work, it is planned to use a wider range of soil samples to confirm the robustness of this method, with a view to developing a rapid, portable and robust system for in-field soil aggregate size distribution assessment based on pattern spectra.

\section{References}

Aitkenhead, M., Donnelly, D., Coull, M., \& Gwatkin, R. (2016). Estimating soil properties with a mobile phone. In A. E. Hartemink \& B. Minasny (Eds.), Digital soil morphometrics (pp. 89-110). Springer International Publishing.

Allen, D. E., Singh, B. P., \& Dalal, R. C. (2011). Soil health indicators under climate change: a review of current knowledge. In B. P. Singh, A. L. Cowie, \& K. Y. Chan (Eds.), Soil health and climate change (pp. 25-45). Springer Berlin Heidelberg.

Ashman, M., Hallett, P., \& Brookes, P. (2003). Are the links between soil aggregate size class, soil organic matter and respiration rate artefacts of the fractionation procedure? Soil Biology and Biochemistry, 35(3), 435-444. 
Bosilj, P., Kijak, E., \& Lefèvre, S. (2018). Partition and inclusion hierarchies of images: A comprehensive survey. Journal of Imaging, 4(2), 33.

Breen, E. J. \& Jones, R. (1996). Attribute openings, thinnings, and granulometries. Computer Vision and Image Understanding, 64(3), 377-389.

Bronick, C. J. \& Lal, R. (2005). Soil structure and management: a review. 615

Ball, B. C., Batey, T., \& Munkholm, L. J. (2007). Field assessment of soil structural quality-a development of the Peerlkamp test. Soil Use and Management, 23(4), 329-337.

Beare, M. H. \& Bruce, R. R. (1993). A comparison of methods for measuring water-stable aggregates: implications for determining environmental effects on soil structure. Geoderma, 56(1-4), 87-104.

Bianconi, F., Di Maria, F., Micale, C., Fernández, A., \& Harvey, R. W. (2015). Grain-size assessment of fine and coarse aggregates through bipolar area morphology. Machine Vision and Applications, 26(6), 775-789.

Bonneel, N., Peyré, G., \& Cuturi, M. (2016). Wasserstein barycentric coordinates: histogram regression using optimal transport. ACM Trans. Graph., $35(4), 71-1$.

Bosilj, P., Aptoula, E., Lefèvre, S., \& Kijak, E. (2016). Retrieval of remote sensing images with pattern spectra descriptors. ISPRS International Journal of Geo-Information, 5(12), 228.

Bosilj, P., Gould, I., Duckett, T., \& Cielniak, G. (2019). Pattern Spectra from Different Component Trees for Estimating Soil Size Distribution. In Burgeth, B., Kleefeld, A., Naegel, B., Passat, N., \& Perret, B. (Eds.), ISMM 2019: Mathematical Morphology and Its Applications to Signal and Image Processing, (pp. 415-427). Springer, Cham. Saarbrücken, Germany.

Geoderma, 124(1-2), 3-22. 
Buscombe, D. \& Masselink, G. (2009). Grain-size information from the statistical properties of digital images of sediment. Sedimentology, 56(2), 421-438.

Cavallaro, G., Falco, N., Dalla Mura, M., \& Benediktsson, J. (2017). Automatic attribute profiles. IEEE T. Image Process., 26(4), 1859-1872.

Chen, Y. \& Dougherty, E. R. (1994). Gray-scale morphological granulometric texture classification. Optical Engineering, 33(8), 2713-2723.

Cousty, J., Najman, L., Kenmochi, Y., \& Guimarães, S. (2018). Hierarchical segmentations with graphs: quasi-flat zones, minimum spanning trees, and saliency maps. Journal of Mathematical Imaging and Vision, 60(4), 479-502.

Czarnes, S., Hallett, P., Bengough, A., \& Young, I. (2000). Root-and microbialderived mucilages affect soil structure and water transport. European Journal of Soil Science, 51(3), 435-443.

Detert, M. \& Weitbrecht, V. (2012). Automatic object detection to analyze the geometry of gravel grains-a free stand-alone tool. In River flow 2012 :

630 Proceedings of the international conference on fluvial hydraulics, (pp. 595600). Taylor \& Francis Group London. San Jose, Costa Rica.

Dougherty, E. R., Pelz, J. B., Sand, F. M., \& Lent, A. (1992). Morphological image segmentation by local granulometric size distributions. Journal of Electronic Imaging, 1(1), 46-61.

635 Doulamis, A., Doulamis, N., \& Maragos, P. (2001). Generalized multiscale connected operators with applications to granulometric image analysis. In Proceedings 2001 International Conference on Image Processing (ICIP), volume 3, (pp. 684-687). IEEE. Thessaloniki, Greece.

Frančišković-Bilinski, S., Bilinski, H., Vdović, N., Balagurunathan, Y., \& Dougherty, E. R. (2003). Application of image-based granulometry to siliceous and calcareous estuarine and marine sediments. Estuarine, Coastal and Shelf Science, 58(2), 227-239. 
Graham, D. J., Reid, I., \& Rice, S. P. (2005). Automated sizing of coarse-grained sediments: image-processing procedures. Mathematical Geology, 37(1), 1-28.

${ }_{645}$ Graham, D. J., Rice, S. P., \& Reid, I. (2005). A transferable method for the automated grain sizing of river gravels. Water Resources Research, 41(7).

Guimarães, R. M. L., Ball, B. C., \& Tormena, C. A. (2011). Improvements in the visual evaluation of soil structure. Soil Use and Management, 27(3), 395-403.

Hadwiger, H. (1950). Minkowskische Addition und Subtraktion beliebiger Punktmengen und die Theoreme von Erhard Schmidt (eng. Minkowski addition and subtraction of arbitrary point sets and the theory of Erhard Schmidt). Mathematische Zeitschrift, 53(3), 210-218.

Heijmans, H. J. A. M. (1999). Connected morphological operators for binary images. Computer Vision and Image Understanding, 73(1), 99-120.

Karunatillake, S., McLennan, S. M., Herkenhoff, K. E., Husch, J. M., Hardgrove, C., \& Skok, J. R. (2014). A martian case study of segmenting images automatically for granulometry and sedimentology, part 1: algorithm. Icarus, 229, 400-407.

Le Bissonnais, Y. (1996). Aggregate stability and assessment of soil crustability and erodibility: I. theory and methodology. European Journal of soil science, 47(4), 425-437.

Maragos, P. (1989). Pattern spectrum and multiscale shape representation. IEEE Transactions on Pattern Analysis and Machine Intelligence, 11(7), 701-716.

Maragos, P. \& Schafer, R. W. (1990). Morphological systems for multidimensional signal processing. Proceedings of the IEEE, 78(4), 690-710.

Maragos, P. \& Ziff, R. D. (1990). Threshold superposition in morphological image analysis systems. IEEE Transactions on Pattern Analysis and Machine Intelligence, 12(5), 498-504. 
Márquez, C. O., Garcia, V. J., Cambardella, C. A., Schultz, R. C., \& Isenhart, T. M. (2004). Aggregate-size stability distribution and soil stability. Soil Science Society of America Journal, 68(3), 725-735.

Matheron, G. (1975). Random sets and integral geometry. Wiley New York.

675 Minkowski, H. (1903). Volumen und Oberfläche (eng. Volume and surface). Mathematische Annalen, 57(4), 447-495.

Monasse, P. \& Guichard, F. (2000). Scale-space from a level lines tree. Journal of Visual Communication and Image Representation, 11(2), 224-236.

Mora, C. F., Kwan, A. K. H., \& Chan, H. C. (1998). Particle size distribution analysis of coarse aggregate using digital image processing. Cement and Concrete Research, 28(6), 921-932.

Pina, P., Lira, C., \& Lousada, M. (2011). In-situ computation of granulometries of sedimentary grains-some preliminary results. J. Coast. Res, 64, 1727-1730.

Ronse, C. \& Heijmans, H. J. A. M. (1991). The algebraic basis of mathematical morphology: II. Openings and closings. CVGIP: Image Understanding, 54(1), $74-97$.

Salehizadeh, M. \& Sadeghi, M. T. (2010). Size distribution estimation of stone fragments via digital image processing. In Bebis, G., Boyle, R., Parvin, B., Koracin, D., Chung, R., Hammound, R., Hussain, M., Kar-Han, T., Crawfis, R., Thalmann, D., Kao, D., \& Avila, L. (Eds.), Advances in Visual Computing, ISVC 2010, (pp. 329-338). Springer. Las Vegas, NV, USA.

Salembier, P., Oliveras, A., \& Garrido, L. (1998). Anti-extensive connected operators for image and sequence processing. IEEE T. Image Process., 7(4), $555-570$.

Salembier, P. \& Wilkinson, M. H. F. (2009). Connected operators. IEEE Signal Processing Magazine, 26(6), 136-157. 
Serra, J. (1983). Image analysis and mathematical morphology. Academic Press, Inc.

Serra, J. \& Vincent, L. (1992). An overview of morphological filtering. Circuits, Systems and Signal Processing, 11(1), 47-108.

Shepherd, T. (2009). Visual soil assessment. volume 1. field guide for pastoral grazing and cropping on flat to rolling country. Horizons Regional Council, Palmerston North, New Zealand, 119.

Silverman, B. W. (2018). Density estimation for statistics and data analysis. Routledge.

Soille, P. (2007). On genuine connectivity relations based on logical predicates. In 14th International Conference on Image Analysis and Processing (ICIAP 2007), (pp. 487-492). IEEE. Modena, Italy.

Soille, P. (2008). Constrained connectivity for hierarchical image partitioning and simplification. IEEE Transactions on Pattern Analysis and Machine Intelligence, 30(7), 1132-1145.

Soille, P. (2013). Morphological image analysis: principles and applications. Springer Science \& Business Media.

Tushabe, F. \& Wilkinson, M. H. F. (2007). Content-based image retrieval using combined $2 \mathrm{~d}$ attribute pattern spectra. In Peters, C., Jijkoun, V., Mandl, T., Müller, H., Oard, D. W., Peñas, A., Petras, V., \& Santos, D. (Eds.), CLEF 200\%: Advances in Multilingual and Multimodal Information Retrieval, (pp. 554-561). Springer, Berlin, Heidelberg.

Urbach, E. R., Roerdink, J. B. T. M., \& Wilkinson, M. H. F. (2007). Connected shape-size pattern spectra for rotation and scale-invariant classification of gray-scale images. IEEE T Pattern Anal., 29(2), 272-285.

Urbach, E. R. \& Wilkinson, M. H. F. (2002). Shape-only granulometries and grey-scale shape filters. In Mathematical morphology: proceedings of the VIth international symposium, ISMM 2002, (pp. 305-314). Sydney, Australia. 
725 Villani, C. (2008). Optimal transport: old and new, volume 338. Springer Science \& Business Media.

Vincent, L. (1993a). Grayscale area openings and closings, their efficient implementation and applications. In Proceedings of EURASIP Workshop on Mathematical Morphology and its Applications to Signal Processing, (pp. 2227). UPC Publications. Barcelona, Spain.

Vincent, L. (1993b). Morphological grayscale reconstruction in image analysis: Applications and efficient algorithms. IEEE transactions on image processing, 2(2), 176-201.

Wendt, P., Coyle, E. J., \& Gallagher, N. (1986). Stack filters. IEEE Transactions on Acoustics, Speech, and Signal Processing, 34(4), 898-911.

Williams, C. K. I. \& Rasmussen, C. E. (2006). Gaussian processes for machine learning, volume 2. MIT Press Cambridge, MA. 